\title{
APRELIMINARY microRNA ANALYSIS OF NON SYNDROMIC THORACIC AORTIC ANEURYSMS
}

\author{
Patuzzo $\mathrm{C}^{1, *}$, Pasquali $\mathrm{A}^{1}$, Malerba $\mathrm{G}^{1}$, Trabetti $\mathrm{E}^{1}$, \\ Pignatti $\mathrm{PF}^{1}$, Tessari $\mathrm{M}^{2}$, Faggian $\mathrm{G}^{2}$
}

\begin{abstract}
*Corresponding Author: Dr. Cristina Patuzzo, Department of Life and Reproduction Sciences, University of Verona, Strada Le Grazie 8, 37134,Verona, Italy; Tel.: +39-45-802-7207; Fax: +39-45-802-7180; E-mail: cristina.patuzzo@univr.it
\end{abstract}

\section{ABSTRACT}

The development of thoracic aortic aneurysms (TAAs) involves a multifactorial process resulting in alterations of the structure and composition of the extracellular matrix (ECM). Recently, modifications in microRNA (miRNA) expression were implicated in the pathogenesis of TAA.

This study presents a preliminary miRNA microarray analysis conducted on pooled ascending aorta RNAs obtained from non familial non syndromic TAA patients (five males and five females) compared to matched control pools. Ninety-nine differentially expressed miRNAs with $>1.5$-foldup- or down-regulation in TAAs compared to controls were identified, $16.0 \%$ of which were similarly regulated in the two sexes.

Genes putatively targeted by differentially expressed miRNAs belonged preferentially to focal adhesion and adherens junction pathways. The results indicate an altered regulation of miRNA-mediated gene expression in the cellular interactions of aneurysmal aortic wall.

Keywords: Adherens junction, Focal adhesion, MicroRNAs (miRNAs), Thoracic aortic aneurysms (TAAs)

1 Department of Life and Reproduction Sciences, University of Verona, Verona, Italy

2 Department of Surgery, University of Verona, Verona, Italy

\section{INTRODUCTION}

Thoracic aortic aneurysms (TAAs) are characterized by a pathological enlargement of the aorta caused by a maladaptive remodeling of the vessel in response to stress and physiological stimuli. The physiological remodeling process within the aortic vascular wall operates to maintain normal aortic function, whereas pathological remodeling can result in excessive degradation of critical extracellular matrix (ECM) components, leading to the loss of mechanical strength and integrity, aortic dilation, dissection, or rupture.

While more than $20.0 \%$ TAAs are inherited as a single gene disorder (e.g., fibrillin-1 gene, FBN1, in Marfan syndrome), the majority of cases are sporadic [1]. The aneurysmal process is now understood to be driven by an unbalanced production of extracellular proteases and inhibitors, but the upstream signalling events are still largely unknown, and especially so for non syndromic events [2]. As recently indicated, an impairment of fine tuning of gene expression in the arterial wall could be related to an altered microRNAs (miRNAs) expression pattern [3].

MicroRNAs are a class of endogenous, small, non coding RNAs regulating the expression of protein-coding genes through pairing with sites in the 3' untranslated region (3'UTR) of their messenger RNA. Due to a perfect or imperfect match, every miRNA may regulate the expression of one 
or more genes, and it is likely that at least $30.0 \%$ of the genes in a cell may be directly regulated by miRNAs [4]. Recent studies have demonstrated that miRNAs are highly expressed in the vasculature and act as important determinants of disease for the cardiovascular system [5]. In particular, two recent studies in humans have reported altered miRNA expression patterns in TAAs and in thoracic aortic dissection after real time-polymerase chain reaction (ReTi-PCR) or by microarray analysis, respectively $[6,7]$. Although not yet fully demonstrated, vascular gene expression is thought to be sex related [8-10].

The aim of this study was to compare the miRNA profiles of ascending aortas from TAA patients and controls by microarray analysis. To check for a possible sex effect, different hybridizations were performed for male and female RNA pools.

\section{MATERIALS AND METHODS}

Patients and Biological Samples. Samples of aneurysmal ascending aortic wall were obtained from 10 patients (five males and five females) affected by non familial non syndromic TAA, during surgical repair of their ascending aneurysms. The syndromic nature of aortic aneurysm has been systematically excluded by careful evaluation of clinical and family history. Patients with aortic dissections, ruptured aneurysms, Marfan syndrome, or other known connective tissue disorders, were excluded from the study. Autoimmune and/or infectious inflammatory diseases, or chest trauma, were also excluded. Control samples of ascending aorta were obtained from 10 heart transplant recipients without aortic aneurysms (five males and five females). All the individuals had a tricuspid aortic valve. Mean age was $66 \pm 9$ years. The study conforms with the principles outlined in the Declaration of Helsinki. Aortic samples were promptly dipped into RNAlater solution (Ambion, Austin, TX, USA) in order to preserve their cellular RNA and maintained at room temperature for 2 hours to facilitate liquid permeation. The samples were then stored at $-80^{\circ} \mathrm{C}$.

Preparation of Microarrays. Total RNA was extracted from the 20 aorta specimens with TRIzol reagent according to the manufacturer's protocol. Total RNA integrity was assessed by an Agilent 2100 Bioanalyzer (Agilent Technologies, Santa
Clara, CA, USA) and RNA integrity numbers (RIN) were sufficient for micro RNA (miRNA) microarray experiments (i.e., RIN >6) [11]. Four samples were then prepared by pooling corresponding RNAs: TAA males, TAA females, control males, and control females.

Sample labeling, PIQOR ${ }^{\mathrm{TM}}$ mirExplore microarray hybridization and fluorescence signal detection were performed by Miltenyi Biotec $\mathrm{GmbH}$ (MACS Service, Köln, Germany). The TAA and control pools were labeled with Hy5 and Hy3, respectively, and competitively hybridized on the same microarray, separately for the two sexes. Fluorescence signals of the hybridized PIQOR ${ }^{\mathrm{TM}}$ Microarrays (Miltenyi Biotec $\mathrm{GmbH}$ ) were detected using a laser scanner from Agilent (Agilent Technologies).

Image and Data Analysis. Mean signal and mean local background intensities were obtained for each spot of the microarray images using the ImaGene $\AA$ software (Biodiscovery, Hawthorne, CA, USA). Low-quality spots were flagged and excluded from data analysis. Unflagged spots were analyzed with the PIQOR ${ }^{\mathrm{TM}}$ Analyzer software (Miltenyi Biotec $\mathrm{GmbH}$ ) that allows automated data processing of the raw data text files derived from the ImaGene software. This includes background subtraction to obtain the net signal intensity, data normalization, and calculation of the Hy5/Hy3 ratios. As an additional quality filtering step, only spots/genes that had a signal higher than the $50.0 \%$ percentile of the background signal intensities were taken into account for the calculation of the $\mathrm{Hy} 5$ / Hy3 ratio.

Up- and Down-Regulated MicroRNA and Gene Pathways. Normalized mean Hy5/Hy3 ratios were determined for four replicas per gene. There was a specific detection even for closely related miRNA family members. MicroRNAs that were $>1.5$-fold-up or down-regulated represented putative candidate miRNAs.

To identify molecular pathways potentially altered by the expression of single or multiple miRNAs, Diana mir- Path Software (Athens, Greece) was used [12]. This web-based application performs an enrichment analysis of multiple miRNA target genes comparing each set of miRNA targets to all known KEGG (Kyoto Encyclopedia of Genes and Genomes, Kyoto, Japan) pathways [13]. 


\section{RESULTS}

The oligonucleotide probes on the microarrays complemented 728 mature miRNAs. Differentially expressed miRNAs ranged from -2.24-fold down regulation to +7.20 -fold up regulation, and are listed in Table 1 . Out of 728 miRNAs detected, 87 resulted differentially expressed in males (11 down- and 76 up-regulated in TAA) and 28 in females (eight down and 20 up-regulated in TAA), compared to their controls. Five and 11 of these miRNAs were down and up regulated in both male and female

Table 1. Ninety-nine differently expressed microRNAs in ascending aortic aneurysm sex-specific RNA pools. Results are indicated as ratios of TAA pool expression (males and females) to their corresponding control pool expression (males or females); blank boxes represent no differential expression. Up-regulated microRNAs have ratios of $>1.5$, while down-regulated microRNAs have ratios of $<0.66$ (if the relative ratio is below 1.0, divide by your ratio to get a fold decrease: $1.0 / 0.66=-1.5$ ).

\begin{tabular}{|c|c|c|c|c|c|c|c|c|}
\hline microRNA & Males & Females & microRNA & Males & Females & microRNA & Males & Females \\
\hline MIR-940 & 0.48 & 0.45 & MIR-370 & 1.64 & & MIR-193A-3P & & 2.25 \\
\hline MIR-923 & 0.56 & & HIV1-MIR-H1 & 1.64 & & MIR-768-5P & 2.27 & \\
\hline MIR-663 & & 0.45 & MIR-383 & 1.64 & & MIR-376C & 2.29 & \\
\hline MIR-133A & 0.56 & 0.48 & MIR-487B & 1.67 & 1.70 & MIR-373 & 2.30 & \\
\hline MIR-133B & 0.56 & 0.50 & MIR-150 & 1.67 & & MIR-765 & 2.32 & \\
\hline MIR-125A-3P & 0.57 & 0.47 & MIR-340-5P & 1.67 & & MIR-493* & 2.34 & \\
\hline MIR-486-5P & 0.57 & 0.57 & MIR-183* & 1.68 & & MIR-422A & 2.35 & 1.56 \\
\hline MIR-145 & 0.61 & & MIR-324-3P & 1.69 & & MIR-23B* & 2.37 & \\
\hline MIR-200B* & 0.62 & & MIR-199A-3P & 1.70 & & $\begin{array}{c}\text { EBV-MIR- } \\
\text { BART13 }\end{array}$ & 2.51 & \\
\hline MIR-193A-5P & 0.63 & & MIR-29B & 1.74 & 1.51 & MIR-17* & 2.64 & \\
\hline MIR-22 & 0.63 & & MIR-222 & & 1.75 & MIR-212 & & 2.46 \\
\hline MIR-665 & & 0.63 & MIR-487A & 1.76 & & MIR-373* & 2.68 & \\
\hline MIR-638 & & 0.63 & MIR-451 & 1.76 & & MIR-520E & 3.76 & \\
\hline MIR-193B & 0.65 & & MIR-210 & & 1.77 & MIR-15B & 2.79 & \\
\hline MIR-146A & 1.51 & & MIR-345-5P & 1.79 & & MIR-25 & 2.80 & 1.66 \\
\hline MIR-150* & 1.52 & & MIR-886-5P & 1.81 & & MIR-26B & 2.84 & \\
\hline $\begin{array}{c}\text { SV40-MIR-S1- } \\
\text { 5P } \\
\end{array}$ & 1.52 & & MIR-874 & 1.82 & & MIR-187* & 2.95 & \\
\hline MIR-23B & 1.52 & & MIR-30.E & 1.82 & & MIR-378 & 2.96 & \\
\hline MIR-425 & 1.54 & & MIR-130B & & 1.88 & MIR-138-2* & 2.98 & \\
\hline MIR-520A-3P & 1.54 & & MIR-421-3P & 1.95 & & MIR-203 & 3.00 & \\
\hline MIR-24-2* & & 1.55 & MIR-520A & 1.97 & & MIR-302C & 3.07 & \\
\hline MIR-376A & 1.56 & & MIR-214* & 1.97 & & MIR-15A & 3.09 & 1.85 \\
\hline MIR-557 & 1.56 & & MIR-188-5P & 1.97 & & MIR-26A-2* & 3.21 & \\
\hline MIR-195 & 1.56 & & MIR-188-3P & & 1.97 & MIR-138-1* & 3.49 & 1.82 \\
\hline MIR-628-3P & & 1.56 & MIR-16-2* & 2.00 & & MIR-223 & 3.53 & \\
\hline MIR-101 & 1.59 & & MIR-16 & 2.02 & & MIR-148A & 3.55 & \\
\hline MIR-675 & 1.59 & & MIR-654-3P & 2.02 & & MIR-221* & 3.76 & \\
\hline MIR-491-3P & & 1.58 & MIR-16-1* & 2.04 & & MIR-146B-5P & 4.00 & 2.75 \\
\hline MIR-532-5P & 1.60 & & MIR-374B & 2.04 & & MIR-21* & 4.21 & 2.46 \\
\hline MIR-518F* & 1.60 & & MIR-34B-3P & 2.13 & & MIR-142-5P & 4.42 & 2.70 \\
\hline MIR-140-5P & 1.60 & & MIR-744 & 2.15 & & MIR-21 & 4.69 & 2.71 \\
\hline MIR-513A-5P & 1.62 & & MIR-185 & 2.17 & & MIR-128 & 4.80 & 2.13 \\
\hline MIR-216A & 1.62 & & $\begin{array}{c}\text { HIV1- } \\
\text { MIR-N367 }\end{array}$ & 2.23 & & MIR-126-3P & 7.20 & \\
\hline
\end{tabular}


TAAs, respectively, for a total of 16 sex-insensitive differentially expressed miRNAs. No miRNA was regulated in the opposite direction in one sex compared to the other.

Table 2 shows the most interesting coordinated gene expression pathways resulting from Diana mirPath software analysis. A multiple testing correction was performed according to Bonferroni's method [14], as indicated in Table 2. Focal adhesion was the most gene enriched pathway. It was putatively targeted by the 11 sex-insensitive up-regulated miRNAs (adjusted $p$ value 5.43E-09). Adherens junction-related genes were also preferentially indicated, putatively targeted by the five sex-insensitive down-regulated miRNAs (adjusted $p$ value 7.12E06 ) and apparently also by the up-regulated miRNAs (adjusted $p$ value 2.56E-04). Another significant pathway was the regulation of actin cytoskeleton (adjusted $p$ value 3.12E-04) that resulted from up-regulated miRNAs analysis.

\section{DISCUSSION}

In this study, RNA pools obtained from ascending thoracic aortic wall fragments of patients affected by TAA were competitively hybridized with control pools on microarrays spotted with oligonucleotides putatively recognizing 728 miRNAs. Ninety-nine miRNAs were differentially expressed. The miRNA sequences listed were matched to gene sequences and linked to annotated pathways of gene expression. The most enriched pathways, which in- cluded a high number of putative target genes of differentially expressed miRNAs, were focal adhesion and adherens junction.

The most interesting result concerned the 11 upregulated miRNAs, both in male and female TAAs, which putatively matched 61 genes related to the adhesion processes. This indicates a significant modulation, i.e., repression, of the focal adhesion pathway. The adherens junction pathway, on the basis of the putative targeting by miRNAs, resulted as repressed and stimulated in 26 and 15 genes, respectively. These opposite signals could be a manifestation of the cellular cohesion impairment and of the attempt to reconstitute the integrity of the aortic wall during the development of TAA.

No sex differences were observed in the present study, as miRNAs differentially expressed in males versus females targeted genes belonging to the same general pathways. Some of the differentially expressed miRNAs identified in this study are in agreement with the literature, as reported below.

Down-Regulated microRNAs. Phenotypic abnormalities of vascular smooth muscle cells (VSMCs) and cardiomyocytes have been observed in MIR-133 knockout mice [15]. MIR-145 is decreased in aortas from patients with an aneurysm and was suggested as a potential biomarker for vascular diseases [16].

Up-Regulated microRNAs. MIR-126, the most up-regulated miRNA in this study, has been implicated in the maintenance of vascular integrity [17] and in vascular cell adhesion molecule expression [18]. MIR-29B-mediated down-regulation of

Table 2. Enrichment analysis results of differentially expressed microRNAs (both in male and female TAAs), obtained by Diana mirPath software.

\begin{tabular}{|c|c|c|c|c|}
\hline $\begin{array}{c}\text { Common } \\
\text { MicroRNAs } \\
\end{array}$ & KEGG Pathways ${ }^{a}$ & $\begin{array}{c}\text { Number of } \\
\text { Genes }^{b}\end{array}$ & $p$ Value $^{c}$ & $\begin{array}{l}\text { Adjusted } \\
p \text { Value }^{\mathrm{d}} \\
\end{array}$ \\
\hline $\begin{array}{l}\text { Down- } \\
\text { regulated (5) }\end{array}$ & $\begin{array}{c}\text { adherens junction } \\
\text { transforming growth factor } \beta \text { signaling } \\
\text { focal adhesion }\end{array}$ & $\begin{array}{l}15 \\
13 \\
21 \\
\end{array}$ & $\begin{array}{l}5.20 \mathrm{E}-08 \\
4.35 \mathrm{E}-04 \\
1.00 \mathrm{E}-03 \\
\end{array}$ & $\begin{array}{l}\text { 7.12E-06 } \\
\text { n.s. } \\
\text { n.s. }\end{array}$ \\
\hline $\begin{array}{l}\text { Up- } \\
\text { regulated (11) }\end{array}$ & $\begin{array}{c}\text { focal adhesion } \\
\text { adherens junction } \\
\text { regulation of actin cytoskeleton }\end{array}$ & $\begin{array}{l}61 \\
26 \\
55\end{array}$ & $\begin{array}{l}3.31 \mathrm{E}-11 \\
1.56 \mathrm{E}-06 \\
1.90 \mathrm{E}-06\end{array}$ & $\begin{array}{l}5.43 \mathrm{E}-09 \\
2.56 \mathrm{E}-04 \\
3.12 \mathrm{E}-04\end{array}$ \\
\hline
\end{tabular}

\footnotetext{
a Pathways with higher enrichment in genes putatively targeted by the miRNAs indicated.

${ }^{\mathrm{b}}$ Number of genes belonging to the pathway and putatively targeted by all the miRNAs indicated.

${ }^{c} p$ Values calculated by input dataset enrichment analysis performed by a Pearson's chi-squared test as indicated by Diana mirPath algorithm [12].

${ }^{\mathrm{d}} p$ Values Bonferroni-corrected for multiple testing.
} 
ECM proteins predisposes the aorta to the formation of aneurysms [19]. MIR-21 has been investigated extensively in various tissues and it has been found to promote vascular smooth muscle cell (VSMC) proliferation [16]. Knockdown of MIR221 and MIR-222 by antisense oligonucleotide miRNA depletion has been foun to reduce VSMCs proliferation in response to vascular injury, and both miRNAs are strongly elevated in vivo in VSMCs following vessel injury [16]. MIR-146, MIR-24 and MIR-26 have been implicated in VSMCs proliferation and contraction [16]. MIR-15A, MIR-16, MIR16-1, MIR-16-2, and MIR-195 belong to a family of miRNAs (the miR-15 family) consistently found to be up-regulated in cardiovascular diseases [5].

In conclusion, and in concordance with other investigators [20], our study indicates that the weakness of the ascending aortic tissue in TAA is linked with a perturbation of cell adhesion and cell interaction gene expression pathways. MicroRNAs probably act as regulators, possibly driving, or at least influencing, the development of the disease.

\section{REFERENCES}

1. Keramati AR, Sadeghpour A, Farahani MM, Chandok $\mathrm{G}$, Mani A. The non-syndromic familial thoracic aortic aneurysms and dissections maps to $15 \mathrm{q} 21$ locus. BMC Med Genet. 2010;11(11):143-148.

2. Barbour JR, Spinale FG, Ikonomidis JS. Proteinase systems and thoracic aortic aneurysm progression. J Surg Res. 2007; 139(2): 292-307.

3. Qin S, Zhang C. MicroRNAs in vascular disease. (Review) J Cardiovasc Pharmacol. 2011; 57(1): 8-12.

4. Lewis BP, Burge CB, Bartel DP. Conserved seed pairing, often flanked by adenosines, indicates that thousands of human genes are microRNA targets. Cell. 2005; 120(1): 15-20.

5. Small EM, Frost RJ, Olson EN. MicroRNAs add a new dimension to cardiovascular disease. (Review) Circulation. 2010; 121(8): 1022-1032.

6. Jones JA, Stroud RE, O'Quinn EC, et al. Selective microRNA suppression in human thoracic aneurysms: relationship of miR-29a to aortic size and proteolytic induction. Circ Cardiovasc Genet. 2011; 4(6): 605-613.

7. Liao M, Zou S, Weng J, et al. A microRNA profile comparison between thoracic aortic dissection and normal thoracic aorta indicates the potential role of microRNAs in contributing to thoracic aortic dissection pathogenesis. J Vasc Surg. 2011; 53(5): 13411349.

8. Sieveking DP, Lim P, Chow RWY, et al. A sex-specific role for androgens in angiogenesis. J. Exp Med. 2010; 207(2): 345-352.

9. Arain FA, Kuniyoshi FH, Abdalrhim AD, Miller VM. Sex/gender medicine - the biological basis for personalized care in cardiovascular medicine. Circ J. 2009; 73(10): 1774-1782.

10. Orshal JM, Khalil RA. Gender, sex hormones, and vascular tone. (Review) Am J Physiol Regul Integr Comp Physiol. 2004; 286(2): R233-R249.

11. Fleige S, Pfaffl MW. RNA integrity and the effect on the real-time qRT-PCR performance. (Review) Mol Aspects Med. 2006; 27(2-3): 126-139.

12. Papadopoulos GL, Alexiou P, Maragkakis M, Reczko M, Hatzigeorgiou AG. DIANA-mirPath: Integrating human and mouse microRNAs in pathways. Bioinformatics. 2009; 25(15): 1991-1993.

13. Kanehisa M, Araki M, Goto S, et al. KEGG for linking genomes to life and the environment. Nucleic Acids Res. 2008; 36(Database issue): D480-D484.

14. Abdi H. Bonferroni and Šidák corrections for multiple comparisons. In: Salkind NJ, Ed. Encyclopedia of Measurement and Statistics. Thousand Oaks: Sage, 2007.

15. Liu N, Bezprozvannaya S, Shelton JM, et al. Mice lacking microRNA 133a develop dynamin 2-dependent centronuclear myopathy. J Clin Invest. 2011; 121(8): 3258-3268.

16. Davis-Dusenbery BN, Wu C, Hata A. Micromanaging vascular smooth muscle cell differentiation and phenotypic modulation. (Review) Arterioscler Thromb Vasc Biol. 2011; 31(11): 2370-2377.

17. Wang S, Aurora AB, Johnson BA, et al. The endothelial-specific microRNA miR-126 governs vascular integrity and angiogenesis. Dev Cell. 2008; 15(2): 261-271.

18. Harris TA, Yamakuchi M, Ferlito M, Mendell JT, Lowenstein CJ. MicroRNA-126 regulates endothelial expression of vascular cell adhesion molecule 1 . Proc Natl Acad Sci USA. 2008; 105(5): 1516-1521.

19. Boon RA, Seeger T, Heydt S, et al. MicroRNA-29 in aortic dilation: implications for aneurysm formation. Circ Res. 2011; 109(10): 1115-1119.

20. Kim JH, Na CY, Choi SY, et al. Integration of geneexpression profiles and pathway analysis in ascending thoracic aortic aneurysms. Ann Vasc Surg. 2010; 24(4): 538-549. 